\title{
EL EFECTO DE LA INFORMACIÓN \\ Y LOS ATRIBUTOS DEL PRODUCTO \\ EN LAS CONDUCTAS DE ELECCIÓN \\ DENTRO DE LA CATEGORIA \\ DE CONSUMO ECOLÓGICO'
}

\author{
Oscar Robayo, \\ Ricardo Guayara, \\ Diana García
}

$\mathrm{n}$ los últimos años se ha logrado
identificar un incremento en el inte-
rés por estudiar las conductas de autocontrol en individuos humanos, en particular aquellas que se relacionan con comportamientos de consumo de bienes tanto tangibles como intangibles. Este interés puede deberse a la preocupación cada vez más palpable tanto en círculos académicos como en sectores gubernamentales con respecto a las muy probables consecuencias negativas que traerán

\section{RESUMEN:}

El presente estudio pretende identificar la manera en que influye un conjunto de variables independientes sobre la conducta de elección en la categoría de productos ecológicos. Específicamente se manipularon cuatro variables: información sobre las consecuencias del consumo, precio de las alternativas, etiqueta ecológica y tiempo de exposición a la información de las marcas. Se diseñaron diez situaciones de elección las cuales variaron de acuerdo con los niveles de cada una de las variables independientes, de tal forma que se generaron 24 condiciones experimentales. Los sujetos respondieron a las condiciones en dos fases, la primera sin información sobre las consecuencias de elección de la marca ecológica y la segunda presentaba una mayor cantidad de información sobre dichas consecuencias, con lo cual se esperaba encontrar una mayor preferencia de los sujetos por dicha marca en la segunda fase. Los resultados indican que los sujetos prefieren en todas las condiciones experimentales la opción ecológica, además el principal factor que demostró influir significativamente en las conductas de elección fue la información sobre las consecuencias. Los demás factores no influyeron de forma significativa. Esto permite concluir que una adecuada y completa información sobre los beneficios de los productos ecológicos conduce al consumidor a asignarle un mayor valor independientemente de que el precio sea igual o mayor al de su competidor no ecológico.

\begin{abstract}
:
The purpose of this study is to identify how a group of independent variables influence the selection behavior in the category of ecologic products. Specifically four variables were taken into account: information on the consequences of consumption, price of the alternatives, ecologic label and time of exposition to the information of the brands. Ten selection situations were designed and they varied depending on the levels of each independent variable, generating 24 experimental conditions. The individuals responded to the conditions in two phases, the first without information on the consequences of the selection of an ecologic brand and the second one included a larger amount of information on these consequences expecting to find a higher level of preference of the individuals for the brand in the second phase. The results indicated that under all the experimental conditions, the individuals preferred the ecologic option, and furthermore the main factor that ended up having the greatest influence on the selection behaviors was the information on the consequences. The other factors didn't have a significant influence. We therefore can conclude that an adequate and complete information on the benefits of ecologic products drives the consumer to give them a higher value, notwithstanding the fact that the price may be the same or higher than the non-ecological competitor's one.
\end{abstract}

1 La investigación fue desarrollada en la ciudad de Bogotá, D.C. durante el primer semestre del 2003. 
los comportamientos inadecuados de los seres humanos relacionados con diversas topografías y funciones de respuesta.

Este interés se ve demostrado en un creciente número de estudios que tratan problemas de conducta tales como hábitos alimenticios inadecuados, cuando las personas consumen grasas saturadas en exceso (Muraven y otros, 1999), o cometen conductas inapropiadas cuando se encuentran bajo los efectos del alcohol (Baumeister y otros, 1994; Hull, 1981), o también, como es el objeto de esta investigación, tienen conductas inadecuadas que afectan directamente al medio ambiente, ya que los consumidores no perciben las consecuencias que su comportamiento ejerce sobre las variables ambientales a lo largo del planeta.

De esta manera, es cada vez más importante tratar de entender cómo las personas llegan a tomar una decisión acerca de qué tipo de producto comprar en función de las consecuencias que cada una de estas acciones de consumo individuales van teniendo de forma acumulativa en los cambios ambientales que se han venido dando.

Para describir porqué un consumidor elige un producto con ciertos atributos en lugar de otro, el modelo preponderante utilizado para explicar la conducta del consumidor ha sido el modelo de la elección racional. De acuerdo con este modelo, los consumidores deciden si deben o no deben realizar una compra y qué deben comprar a través de la comparación de los costos y beneficios de las alternativas (Mowen, 1998). Desde este punto de vista, este mismo autor define al consumidor como un ser libre de emociones que evalúa las alternativas de forma sistemática sin mayores variaciones a lo largo del tiempo. Sin embargo, como lo sugieren Hoch y Loewenstein (1991), una visión que proporcione un mayor entendimiento del consumidor debe reconocer que las personas son influidas tanto por consecuencias a largo plazo que requieren un pensamiento más racional, como por consecuencias a más corto plazo influidas por factores más emocionales.

Estos dos estilos de elección del consumidor han sido estudiados tanto en forma separada como a través de su interacción. Sin embargo, se puede notar que el modelo racional ha sido elaborado de una manera mucho más formal a través de funciones matemáticas mientras que el modelo hedonista-emocional ha sido descrito de una forma más cualitativa (Hoch y Loewenstein, 1991).

Dentro de los modelos integrales propuestos más recientemente para explicar estos comportamientos, se encuentra en primer lugar, el modelo basado en el concepto económico del descuento del valor de las alternativas, planteado inicialmente por Ainslie (1975), el cual ha influido a muchos teóricos e investigadores contemporáneos como se verá más adelante. De acuerdo con este punto de vista, las personas se ven atraídas de forma desproporcionada por las alternativas que ofrecen una recompensa más inmediata, en tanto que las alternativas que ofrecen una recompensa más lejana en el tiempo van sufriendo de un descuento en su valor percibido por parte del individuo.

Por otra parte, este tipo de comportamientos relacionados con la elección de alternativas que se diferencian, en primer 
lugar, por las consecuencias que generan tanto para el individuo como para la sociedad en su conjunto, y en segundo lugar, por el período de tiempo que transcurre entre la elección y la aparición de dichas consecuencias, han sido estudiados dentro del marco del Análisis Experimental del Comportamiento bajo el modelo de autocontrol.

El modelo de autocontrol surge de la literatura existente sobre la ley de igualación, un modelo cuantitativo de la conducta de elección. Más específicamente, el autocontrol se define de acuerdo con este modelo como la elección de una alternativa de acción que proporciona una consecuencia más grande y demorada, en lugar de una alternativa que ofrece una consecuencia más pequeña e inmediata (alternativa impulsiva. Logue, 1996).

La presente investigación se centra en evaluar las conductas de elección del consumidor dentro de la categoría de productos ecológicos ante varias alternativas que difieren en diversos atributos o características. Por un lado se encuentran los productos ecológicos, y por el otro, los pro- ductos tradicionales o no ecológicos, los cuales generan diferentes consecuencias tanto para el individuo como para el medio ambiente. Dichas consecuencias serán tratadas en detalle más adelante.

En este sentido, es importante acudir al "modelo de autocontrol" formulado por Logue (1995), el cual describe que las conductas de autocontrol e impulsividad están en función de dos factores: el tamaño de la consecuencia y la cantidad de demora de esta. En general, la investigación dentro de la psicología experimental ha demostrado que:

1. Cuando la demora para una recompensa grande se incrementa, al mismo tiempo disminuye el autocontrol.

2. Es más probable que las personas esperen por una consecuencia más grande que por una recompensa más pequeña.

Dentro del campo de la psicología del consumidor también se han llevado a cabo múltiples investigaciones que han tenido como objetivo central el estudio del autocontrol en diversas situaciones de consumo. Dentro de las más importantes se encuentran las investigaciones sobre
Los humanos y otros animales incorporaron dentro de su repertorio una característica particular de la conducta: una tendencia a descontar, es decir, a darle menos valor a las consecuencias demoradas, una tendencia que causa impulsividad.

\section{RESEÑA DE LOS AUTORES:}

Oscar Javier Robayo es psicólogo, especialista en Psicología del Consumidor. Actualmente se desempeña como investigador independiente y es docente de las materias de Comportamiento del Consumidor, Investigación de Mercados I, e Investigación de Mercados II en la Facultad de Mercadeo y Publicidad del Politécnico Grancolombiano.

Ricardo Guayara Piragauta ha realizado estudios en Mercado y Ventas, Psicología y es especialista en comportamiento del consumidor. Ha trabajado en empresas como Fundalectura, Pomona- Cadenalco, Danzas y Sky TV. También se desempeña como docente del Politécnico Grancolombiano y otras prestigiosas universidades.

Diana Lucía García es mercadóloga, especialista en Psicología del Consumidor. Ha desempeñado importantes cargos dentro de la organización Éxito-Cadenalco y actualmente trabaja como ejecutiva en la empresa Impulso y Mercadeo. 
compradores compulsivos, como la realizada por O'Guinn y Fabers (1989), la cual concluyó que este tipo de compradores prefería la alternativa impulsiva (comprar de forma exagerada productos que en realidad no tenían ningún valor en su vida diaria) no tanto por los productos en sí mismos, sino por el placer de comprarlos. En otras palabras, la consecuencia que mantiene las compras impulsivas es el placer que se genera en el momento de comprar estos artículos.

Otra línea de investigación en autocontrol muy reciente es la que han trabajado Kivetz y Simonson (2002), en relación con las conductas de trabajo y ocio. A partir de sus estudios estos autores han conceptualizado el exceso de trabajo como una falla en la autorregulación, es decir, que preferir seguir trabajando continuamente, en lugar de tomar descansos regulares, puede ser visto como una conducta impulsiva, ya que a largo plazo, el exceso de trabajo conlleva a graves problemas psicológicos y de salud. Por lo tanto, después de un largo tiempo de trabajo, la opción de autocontrol consiste en tomar un descanso, ya que esta opción brinda la mejor consecuencia a largo plazo: tener un estilo de vida más saludable.

Como lo demuestran los estudios que se acaban de citar, el que se lleve a cabo una conducta de autocontrol o de impulsividad depende del contexto en el cual se dé la elección. Una respuesta de autocontrol es aquella que resulta de una consecuencia más grande y más demorada, mientras que una respuesta impulsiva ocurre de forma contraria. El contexto puede afectar también una respuesta que es clasificada como autocontrolada o impulsiva, que afecta el grado con el cual un individuo valora una recompensa en particular.

Los humanos y otros animales incorporaron dentro de su repertorio una característica particular de la conducta: una tendencia a descontar, es decir, a darle menos valor a las consecuencias demoradas, una tendencia que causa impulsividad. Sin embargo, es posible demostrar que descontar las consecuencias demoradas (darles menos valor), a menudo ha sido adaptativo para los otros animales que evolucionaron y que este rasgo o característica ya no es tan adaptativo para los seres humanos.

Las demoras en los eventos en algunas clases de ambientes primitivos, era poco probable que ocurrieran, por lo que esperar por estas consecuencias no era lo más beneficioso. En otras palabras, la impulsividad y no el autocontrol, probablemente haya maximizado todos los beneficios en un ambiente en el cual los eventos futuros eran inciertos. La impulsividad involucra una asignación de un menor valor para las consecuencias demoradas. Por lo tanto, es probable que durante la evolución, los seres humanos y otros animales que tendieron a darle menos valor a las consecuencias demoradas que a las inmediatas hayan sobrevivido con mayor probabilidad, al menos en algunas situaciones.

El medio ambiente actual de la mayoría de las personas es muy diferente al de los cazadores - recolectores o al de las sociedades agrícolas primitivas. Primero, para la mayoría de la gente de hoy, la comida de alguna manera está siempre disponible. Segundo, la expectativa de vida hoy en día es considerablemente más amplia que la de los antepasados humanos. 
Tercero, en la cultura actual se han formalizado las reglas con las cuales la gente debe vivir. Cuarto, ahora se tiene más conocimiento sobre la probabilidad de ocurrencia de ciertos eventos futuros tales como: clases particulares de tiempo meteorológico, tendencias demográficas y de consumo $y$, en particular para el presente estudio, la probabilidad de que se agoten los recursos naturales mundiales como consecuencia de los hábitos de consumo y estilos de vida actuales. Dado que muchos eventos futuros en el medio ambiente actual son altamente predecibles, no atender a estos eventos puede llegar a ser muy desadaptativo.

A partir de la investigación en esta área se han descubierto una serie de factores que son responsables del desarrollo del autocontrol. Algunos de estos factores podrían ser clasificados como factores biológicos y algunos como factores medioambientales. En general, se ha encontrado que el autocontrol aumenta con la edad y la experiencia en diferentes tipos de actividades. Así mismo, hay mayor autocontrol cuando las elecciones se relacionan con bienes que implican una gran duración en el consumo, como por ejemplo, un electrodoméstico, en tanto que aquellos bienes que se consumen más rápido pueden facilitar una respuesta más impulsiva (Rachlin, 2000). Este hecho se puso a prueba en el presente estudio mediante la inclusión de productos de mayor duración en su consumo (Ej.: lavadora y secadora) y productos que requieren un menor tiempo de consumo (Ej.: esponjillas, detergentes, etc. ).

Estas definiciones de autocontrol e impulsividad son muy similares a las definiciones dadas por Baumeister (1999) de autorregulación y falla en la autorregulación, respectivamente. De acuerdo con Baumeister (1999), la autorregulación ocurre cuando alguien "siente un impulso para actuar en una forma que va en contravía de sus estándares de conducta deseable" (pág. 436). En contraste, "La falla en la autorregulación significa actuar de acuerdo a ese impulso que viola sus standards de conducta" (pág. 436). Al comparar estas definiciones con las dadas anteriormente se encuentra que los estándares de la persona pueden ser vistos como la alternativa más grande y demorada (autocontrol), mientras que el "impulso" es visto como la alternativa más pequeña e inmediata (impulsividad). En otras palabras una persona tiene que elegir entre la falla en la autorregulación (impulsividad) y la autorregulación (autocontrol). Por lo tanto, el modelo de Baumeister y el modelo de Logue se refieren a los mismos fenómenos conductuales.

Otra teoría, denominada Teoría de Limitación Temporal planteada por Liberman y Trope (1998), sugiere que las consecuencias que se encuentran distantes en el futuro, el consumidor las percibe en un nivel más alto (más abstracto) que las consecuencias más cercanas temporalmente. En particular, las decisiones con respecto a situaciones que se encuentran distantes en el futuro se ven influidas por consideraciones de deseabilidad de la consecuencia, mientras que las decisiones sobre situaciones cercanas en el futuro se ven influidas por motivos más prácticos y concretos (Kivetz y Simonson, 2002). Estos resultados sugieren que cuando una alternativa ofrece una consecuencia que es psicológicamente menos concreta (como el mejoramiento ambiental, por ejemplo), los sujetos, con mayor probabilidad, 
escogerán la alternativa autocontrolada. Con respecto al consumo de productos ecológicos, se podría afirmar que la elección de la alternativa autocontrolada tendría unas consecuencias lejanas a nivel temporal, por lo cual podría ser más probable que el consumidor escoja el producto ecológico, ya que la consecuencia de esa decisión sería psicológicamente menos concreta de acuerdo con esta teoría. Además, la elección de una marca ecológica es más deseable para las personas cuando se piensa en las consecuencias a largo plazo, lo cual hace que estas opciones tengan una mayor probabilidad de ser elegidas. Las implicaciones que este planteamiento tiene para la presente investigación se tratan en la discusión de los resultados.

Por otra parte, el problema del autocontrol fue examinado por Schelling como parte de un área mayor que él denominó "egonomía" o "la economía del sí mismo" (Schelling, 1984). Este autor concluyó que existía un problema de miopía del autocontrol como resultado de los esfuerzos hechos por el consumidor para restringir, en un primer momento, su conducta futura y sus alternativas de elección. Esta conducta se denomina compromiso previo y hace parte de lo que Schelling denomina "autocomando anticipatorio". Para explicar este compromiso previo, el autor evoca la noción de los múltiples patrones de compra que existen en diferentes momentos para un mismo individuo. De acuerdo con Schelling, una persona puede tener un patrón de consumo conservador y previsor que puede anticipar; por ejemplo, que no va a resistirse de tomar unos tragos en una fiesta. Por lo tanto, este patrón previsor hace que se in- cremente el costo de consumir alcohol por medio del compromiso previo con la abstinencia. Este compromiso comprendería tomar el medicamento "Antabuse" el cual produce nausea y vomito si se llega a tomar alcohol.

Otra forma de autocontrol es planteada por Thaler y Shefrin (1981), la cual consiste en limitar a un número reducido de alternativas que el consumidor debe escoger. Por ejemplo, si una persona va a comprar un televisor y se caracteriza por ser impulsiva, el compromiso previo consistiría en determinar de antemano un máximo de tres alternativas para escoger que impliquen una solución viable a su necesidad. De esta forma estaría evitando llegar al punto de venta y dejarse guiar por las claves periféricas, las cuales lo inducirían a tomar una elección impulsiva.

Relacionado con lo anterior, Ainslie (1975), a través del modelo del descuento del valor, explica este problema de la miopía del autocontrol utilizando la función hiperbólica de descuento temporal, la cual predice que la preferencia por recompensas demoradas, pero más grandes frente a recompensas pequeñas e inmediatas se revertirá en función de la proximidad temporal de dichas recompensas. Por lo tanto, muchos consumidores emplean un mecanismo de compromiso previo que limite de forma irreversible la conducta futura de manera que la alternativa impulsiva no pueda ser obtenida cuando esté mucho más próxima en el tiempo y sea, por lo tanto, más tentadora para la persona. Ejemplo, cuando una persona programa un reloj despertador en la noche para asegurarse de que se va a despertar a tiempo en la mañana. 
Otro modelo de autocontrol es planteado por Rachlin (2000), y se fundamenta en lo que él ha concebido como conductismo teleológico. Esta concepción plantea que la mente de una persona se forma a partir de los patrones de elección que se presentan a lo largo de un período de tiempo. A través de estas elecciones se va generando una ambivalencia que puede definirse como "la coexistencia dentro de una persona de pensamientos y emociones opuestos dentro de un mismo contexto". Estos sentimientos opuestos generan elecciones opuestas: las personas son ambivalentes, ya que cambian sus elecciones de un momento a otro, para escoger una alternativa ahora y otra más adelante. La indecisión y la duda son signos de una ambivalencia subyacente. Pero la ambivalencia por sí misma sólo se puede manifestar a través de la elección de las dos alternativas, una en un momento, y la otra en un momento diferente.

Con respecto al problema de consumo ecológico se debe tener en cuenta este hecho, ya que los consumidores no van a escoger siempre la alternativa ecológica y tampoco van a escoger siempre la no ecológica. Es decir, que con una consideración más de tipo molar que molecular, las elecciones tendrán alguna variación. Uno de los objetivos de esta investigación consiste precisamente en identificar el nivel de consistencia de las elecciones en dos momentos diferentes en el tiempo. Este autor también plantea que puede existir una ambivalencia simple (por ejemplo, escoger entre un cono de chocolate o uno de vainilla), la cual es común a todas las personas y no representa un problema de autocontrol. Para el caso de los productos ecológicos cabe pregun- tarse si los consumidores consideran que la elección entre dos alternativas (por ejemplo, esponjilla ecológica y no ecológica) puede llegar a generar una ambivalencia simple, en la que no exista mucho compromiso para evaluar las consecuencias futuras de cada una de estas dos opciones. Puede ser que al estar al frente de estas dos opciones, la persona no tenga una percepción abstracta y vaga de las consecuencias y no se vea forzada a evaluar con mayor profundidad los atributos, debido a que el ambiente no le ofrece una retroalimentación lo suficientemente notoria que le permita valorar las consecuencias en un momento específico del tiempo.

$\mathrm{Al}$ contextualizar la situación de ambivalencia simple dentro de la elección del consumidor, se concluye que en realidad sí existe un problema de autocontrol ya que la elección de la marca ecológica es objetivamente deseable en cualquier momento. En otras palabras, no existen momentos donde sea bueno consumir la ecológica y otros donde no lo sea. Por lo tanto, una inconsistencia muy fuerte o una preferencia por la marca no ecológica sí representa un problema de autocontrol que pone en peligro la sostenibilidad de la sociedad de consumo tal como existe hoy día.

Como lo demuestra el estudio llevado a cabo por la firma Roper International Research Associates en 1995 (ver referencias), la preocupación mundial por los problemas ambientales viene en aumento. Este estudio tuvo una muestra de 30.000 adultos en más de cuarenta países en los cinco continentes, y encontró, entre otros hallazgos importantes, que un $25 \%$ de la muestra global creía que la situación ambiental de su país se encontraba muy cer-
Otro dato concluyente sugiere que el $64 \%$ del total de la muestra considera que "la protección del ambiente es el principal problema que debe tratarse, incluso a expensas del crecimiento económico". 
ca del peor nivel posible, en contraste con un $8 \%$ que pensaba que la situación ambiental era la mejor. También se encontró que las zonas geográficas donde existe un mayor pesimismo sobre el desarrollo de estos temas son, en primer lugar, los países de la antigua Unión Soviética, América Latina y los países de Oriente Medio. Otro dato concluyente sugiere que el 64\% del total de la muestra considera que "la protección del ambiente es el principal problema que debe tratarse, incluso a expensas del crecimiento económico".

Por otra parte, Rachlin plantea un segundo tipo de ambivalencia, llamada ambivalencia compleja. En este tipo de situaciones la persona no tiene una conciencia clara de las consecuencias de su comportamiento. Por ejemplo, un alcohólico nunca esta libre del deseo de tomar excepto quizás por un breve periodo de tiempo después de una resaca; incluso en ese momento es probable que quiera curar dicha resaca tomando más alcohol. El alcohólico está continuamente asediado por la ambivalencia; puede tomar en un momento y abstenerse en otro, e incluso, cuando se abstiene, siente la tentación de tomar. Este patrón corresponde a la ambivalencia compleja. A diferencia de la ambivalencia simple no se puede evitar con mecanismos de compromiso sencillos, como en el caso del reloj despertador mencionado anteriormente. Además, en la ambivalencia compleja la alternativa de autocontrol no se percibe de forma clara (se concibe como algo difuso y abstracto), no tiene un periodo de aparición definido, lo que hace que se presente una mayor preferencia por una alternativa impulsiva. De otro lado, en la ambivalencia simple, las alternativas y sus consecuencias son clara- mente identificables y medibles por el consumidor y su tiempo de aparición está muy bien definido.

Como se desprende de lo anterior, las situaciones de elección en la presente investigación se ajustan a un contexto de ambivalencia compleja, ya que las consecuencias de la alternativa ecológica son difusas y difíciles de percibir para el consumidor típico, sumado a una aparición temporal no definida de la consecuencia; por ejemplo, al tener la alternativa de escoger unos platos desechables ecológicos sin tener claro el tiempo de la biodegradación de los mismos e, incluso, el impacto positivo que pueden causarle al ecosistema y las consecuencias a mediano y largo plazo.

Otra característica de la ambivalencia compleja consiste en que cada vez que la persona tiene que elegir, por ejemplo, entre comerse un helado de chocolate y guardar la línea para no ser obeso, la alternativa impulsiva (helado) tiene un valor local mucho mayor que la alternativa relacionada con no aumentar de peso, la cual se encuentra aún muy lejos y se percibe como difusa para la persona. De esta manera, la persona elige de acuerdo con el valor inmediato que le ofrezcan las alternativas: un helado es claramente perceptible y sus efectos son muy placenteros en el momento presente, en tanto que tener un peso adecuado y ser saludable no tiene un momento de aparición preciso, con lo cual el valor de esta opción, en ese momento de la elección, es menor al de la alternativa impulsiva.

En resumen, la ambivalencia se presenta debido a que, si se le pregunta a una persona cuál es su preferencia en cuanto al sostenimiento del medio ambiente, ésta 
probablemente dirá que es más adecuado tener conductas positivas (como comprar productos con sello verde, reciclar los desechos, etc.), que conduzcan a un resultado agradable a largo plazo para todos. Sin embargo, en cada una de las pequeñas elecciones que van conformando su patrón de comportamiento (incluido el consumo de bienes), la persona puede valorar más un producto económico que no tenga sello verde, frente a otro que sí lo tiene y que es más costoso.

Esta dificultad para valorar la alternativa autocontrolada (ecológica) se refleja en las tendencias de comportamiento que han investigado en países industrializados autores como Ottman (2001). Este autor argumenta que, en la mercadotecnia convencional, las variables demográficas se relacionan con la intención de compra de ciertos productos, pero en la mercadotecnia verde lo que determina la intención de comprar productos ecológicos son las creencias del consumidor de poder hacer la diferencia en estos aspectos, algo que se conoce como empoderamiento. La investigación ha comprobado que la variable predictora más precisa para saber la probabilidad de que un individuo pague más por una fuente de energía renovable no es la educación ni el ingreso, sino el ser miembro (o contribuir con dinero) de algún grupo ambientalista. En Colombia las condiciones culturales no han permitido la generación y consolidación de grupos ambientalistas, con lo cual se dificulta el fortalecimiento de los valores ecológicos dentro de las prácticas culturales, y da como resultado un nivel mínimo de conductas ecológicamente conscientes. En Estados Unidos los miembros de este tipo de grupos son sorprendentemente diversos ya que incluyen tanto a profesionales que viven en centros urbanos como a familias de sectores rurales.

Los niveles de involucramiento y empoderamiento varían mucho dentro de la población. Un proceso de segmentación de los consumidores americanos llevado a cabo por la Roper International Research Associates (1995), identifica que un 15\% de población con alto nivel educativo dice estar dispuesta a pagar un precio mayor o a renunciar a ciertas comodidades para asegurar un ambiente más limpio. También identifica a un $37 \%$ del público que admite no ser ambientalista, pero más por razones de indiferencia que por creencias antiambientalistas. En medio de estos dos segmentos se encuentran los indecisos que dicen ser ambientalistas, aunque no actúan completamente de acuerdo con sus reportes verbales. Esta compañía ha hecho un seguimiento a estos segmentos desde 1990, y en 1996 estableció la presencia de cinco segmentos, los cuales se resumen a continuación.

Estos segmentos corresponden, en primer lugar, a los "azules verdaderos" (True Blues), con el $10 \%$ de la población norteamericana, que se caracterizan por tener fuertes creencias ambientales y viven de acuerdo con ellas. Son los ambientalistas más comprometidos, ya que creen que ellos personalmente pueden hacer una diferencia en la solución de los problemas planetarios. Son política y socialmente activos y dedican mucho tiempo y energía en prácticas ambientalmente seguras, tratando de influir con ello a otras personas para que hagan lo mismo. De los cinco grupos están entre las personas con ma- 
yor educación y tienden a ser mujeres blancas que viven en el medio oeste o en el sur de los Estados Unidos.

En segundo lugar, se cuentan "los medio verdes” (Greenbacks), que representan sólo el 5\% de la población americana y se caracterizan por su deseo de pagar un precio extra por obtener productos ambientalmente seguros. Pueden llegar a pagar hasta un $22 \%$ más por un producto verde. Se preocupan por el ambiente, pero se sienten muy ocupados en otras labores como para dedicar tiempo a actividades en grupos ambientalistas. Al igual que el grupo anterior, sus compras de productos verdes están por encima del promedio de todo el país. Tienden a ser hombres blancos, casados, que viven en el medio oeste $(35 \%)$ y en el oeste $(24 \%)$, tienen buen nivel educativo, son jóvenes, con edad media 37 años y están en empleos medios.

El tercer grupo corresponde a los Aspiracionales (Sprouts) comprenden el 33\% de la población. Quieren involucrase en actividades ambientales de vez en cuando, pero sólo si se requiere un esfuerzo pequeño. Por esto, el reciclaje es su actividad ambiental más común. Se fijan en las etiquetas de los productos verdes, aunque no tanto como los grupos anteriores. Su preferencia frente a los productos verdes se reduce dramáticamente cuando estos son más costosos que sus competidores no ecológicos. Cuando compran productos ecológicos, sólo pagan un $4 \%$ extra frente a las otras marcas. La mayoría de estas personas son mujeres con una edad media de 43 años y se distribuyen en todo el país. Tienen buen nivel educativo y cerca de la mitad están casadas.
El cuarto grupo esta conformado por los inconformes, grousers, representan el $15 \%$ de la población. Estas personas no creen que los individuos puedan hacer algo para proteger el ambiente. En lugar de ello creen que la responsabilidad es del gobierno y de las grandes empresas. No están bien informados sobre los problemas ambientales y menos de la mitad recicla botellas y latas regularmente, aunque muchas veces lo hacen de forma inadecuada. Realizan el reciclaje más por cumplir las leyes locales que por contribuir a mejorar el ambiente. Según ellos, están muy ocupados para realizar labores ambientales y creen que los productos verdes son demasiado costosos y no funcionan también como las marcas tradicionales. Demográficamente son similares al promedio nacional, aunque con una proporción mayor de miembros afroamericanos.

Para el caso colombiano sería oportuno establecer qué porcentaje de la población correspondería a este segmento ya que en el país no existe información sobre estudios en esta área. Es decir, se debe establecer si existe un compromiso personal con respecto al bienestar ambiental, o si el consumidor puede llegar a pensar que la responsabilidad es de otros, por ejemplo, del gobierno de turno.

Finalmente, de acuerdo también con la firma Roper International Research Associates (1995), están los compradores básicos, basic browns. Estos consumidores son el grupo más grande; representan el 37\% de la población. Ellos, simplemente, no están convencidos de que los problemas ambientales sean del todo serios. No buscan excusarse por su inactividad frente al 
tema ambiental, ya que ni siquiera les interesa. Muy pocos reciclan y sólo el 3\% de ellos compran productos reciclados. Este grupo tiene el ingreso medio más bajo, con el más bajo nivel de educación y se concentran desproporcionadamente en el sur del país. De nuevo, sería interesante establecer a través de un esfuerzo investigativo de gran alcance la distribución de la población colombiana con respecto a este tipo de conductas, es decir, establecer qué perfil se ajusta al de un comprador consciente ambientalmente y qué perfil correspondería a un comprador más "básico", menos comprometido con las conductas ambientalmente seguras, con los respectivos porcentajes frente al total de la población.

Teniendo en cuenta los conceptos planteados anteriormente, la presente investigación intenta determinar el nivel en que influyen las variables del producto en el proceso de elección (autocontrol vs. impulsividad) del consumidor para la categoría de consumo ecológico. Se espera encontrar si el consumidor prefiere las marcas con características ecológicas o si por el contrario, prefiere las marcas tradicionales que no poseen estas características. Otro objetivo consiste en identificar si el consumidor colombiano demuestra algún nivel de interés por estos nuevos productos que ya se han desarrollado en muchos países avanzados, sobre todo en Europa y también en los Estados Unidos.

Los resultados del estudio pueden ser utilizados por todas aquellas compañías que tengan una filosofía de sostenimiento ambiental a largo plazo que se vea reflejada en su estrategia de negocio. Esto incluye sus productos, su comunicación y orientación hacia el consumidor y, en general, su responsabilidad social frente a la humanidad.

Es crucial, por lo tanto, identificar las variables que pueden influir en los consumidores para que escojan aquellos productos que generen una consecuencia positiva en la protección y sostenimiento del medio ambiente a largo plazo, frente a las alternativas que a corto plazo pueden ser más valoradas por el consumidor, pero que causan, al mismo tiempo, consecuencias acumulativas devastadoras para el bienestar del medio ambiente y, por ende, de la humanidad entera.

\section{USUARIOS DIRECTOS E INDIRECTOS DE LA INFORMACIÓN}

Los usuarios directos son las compañías que en este momento trabajan con productos de categoría ecológica y buscan dar una solución ambiental a los usuarios indirectos, es decir, al consumidor final tanto actual como potencial. También el gobierno central se puede favorecer al facilitar la generación de estrategias que se encuentren en un nivel permaclon (manejo de metacontingencias que cambien las prácticas culturales dañinas por prácticas ambientalmente sostenibles a largo plazo en toda una sociedad).

\section{DEFINICIÓN DE VARIABLES}

VARIABLES INDEPENDIENTES

Información sobre consecuencias de la elección: Consiste en la información por escrito que se le dará a los sujetos en el momento de la evaluación de alternativas. Esta información describe si el producto ofrece una consecuencia positiva ambientalmente y más tangible que pueda llegar a influir en la elección del consumidor.
Se espera encontrar si el consumidor prefiere las marcas con características ecológicas o si por el contrario, prefiere las marcas tradicionales que no poseen estas características. 
La información se manipuló en dos niveles. En una primera fase, se dio información básica de los productos sin profundizar en los efectos ambientales. En la segunda fase, en cambio, se añadió información sobre los efectos positivos de la marca ecológica, al tiempo que se dio más información sobre los atributos y desempeño de la marca no ecológica. En las dos fases, la cantidad de información fue equilibrada, de tal forma que la cantidad de información no influyó a favor de ninguna opción de respuesta.

Precio: Es el valor monetario en pesos colombianos que tuvieron las diferentes alternativas de elección. De acuerdo con Solomon (2001), el precio es la asignación de valor a un producto; es el valor que los clientes dan o intercambian para obtener un producto deseado. Todas las alternativas, en todos los estudios, tuvieron un precio visible que se modificó de acuerdo con el objetivo de cada experimento. De acuerdo con esto, se manejaron tres niveles de precio. En el primer nivel, las dos alternativas tuvieron un precio igual, acorde con el precio promedio del mercado. En el segundo nivel, la alternativa ecológica tuvo un precio $20 \%$ mayor a la alternativa no ecológica. Y en el tercer nivel, la alternativa ecológica tuvo un precio $20 \%$ mejor que la otra.

Etiqueta: Es la comunicación de mercadotecnia que aparece impresa en la cubierta o recipiente de un producto, (Solomon 2001). Para las alternativas de elección se incluyó el nombre de marca como marca $A$, marca $B$, etc. $Y$ se mantuvo la información sobre beneficios del producto, advertencias, indicaciones sobre el uso y, lo más importante, los sellos ecológicos.
Consistente con el marco teórico, se considera que la etiqueta ecológica puede llegar a funcionar como un estimulo discriminativo que induzca al comprador a adquirir la alternativa autocontrolada. Por lo tanto, en la mitad de las situaciones de elección, la alternativa ecológica se presentó junto con un sello verde y la otra mitad se presentó sin el sello verde.

Tiempo de evaluación: Es el número de segundos en que las dos alternativas estuvieron expuestas a los sujetos. En cualquier momento, dentro de este período, el sujeto realizó su elección. En esta variable se escogieron dos condiciones de presentación. En la primera, los sujetos tuvieron 60 segundos como máximo para realizar su elección, en tanto que en la segunda condición, los sujetos tuvieron solo 30 segundos como máximo para la elección.

\section{VARIABLES DEPENDIENTES}

Elección de consumo: Es la ejecución de un comportamiento frente a dos alternativas: una de autocontrol y la otra impulsiva. Esta variable fue medida a través de la marcación de una entre dos teclas del computador, cada una representa una alternativa. Estos datos, fueron analizados de forma estadística posteriormente.

\section{DEFINICIÓN DE HIPÓTESIS:}

$\mathrm{H} 1$ : Cuando el consumidor se enfrenta a una situación de elección en la que existe una alternativa ecológica, que es más costosa, frente a otra opción más económica, pero no ecológica, el sujeto preferirá esta última (alternativa impulsiva).

$\mathrm{H} 2$ : El consumidor elegirá aquella alternativa que posea un sello ecológico frente a otra alternativa que no lo posea. 
H3: Cuando el consumidor tiene un período de exposición a los estímulos mayor, escogerá con mayor probabilidad la alternativa que implica autocontrol.

$\mathrm{H} 4$ : En el caso en que se le brinde información concreta sobre las consecuencias de la elección de la alternativa autocontrolada, el consumidor elegirá esta opción con una mayor probabilidad.

\section{MÉTODO}

Diseño: Para los diferentes estudios se aplicó un diseño cuasiexperimental, ya que los sujetos no fueron asignados aleatoriamente a los grupos, pero sí se mantuvieron las demás condiciones de los diseños experimentales, es decir, el control y manipulación de las variables independientes y la medición del efecto sobre la variable dependiente (Hernández y otros, 1991).

Se utilizo un diseño factorial $(3 \times 2 \times 2)$ anidado en la variable "información sobre las consecuencias de la decisión”. Se escogió este diseño ya que permite dividir el estudio en dos grandes fases que involucran principalmente la condición de ausencia y presencia de información. De esta forma, en cada una de las dos fases se combinan todas las condiciones de las tres variables restantes. A continuación se describe en detalle la estructura del diseño para la presente investigación. (Ver tabla No.1).

El diseño factorial ha sido anidado para las dos condiciones de la variable "información de consecuencias de la decisión". En una primera fase se aplicó a los sujetos la condición que establece la ausencia de información sobre las consecuencias que tendría la elección de la alternativa autocontrolada, mientras que en la segunda $f a-$ se se le dio a los mismos sujetos la información adecuada acerca de los posibles efectos en el medio ambiente que presenta la elección de dicha alternativa. Cada individuo estuvo expuesto a las mismas condiciones de precio, etiqueta y tiempo de exposición. El único cambio entre la primera y la segunda fase fue la cantidad de información suministrada sobre los atributos de los productos.

En el análisis de resultados, se establece si existe o no, un cambio en la decisión entre la primera y la segunda fase, de tal forma que la alternativa ecológica sea más preferida en la segunda fase debido a la información recibida sobre las consecuencias favorables que ofrece esta opción de compra.

En segundo lugar, se ha establecido como segunda variable independiente la presencia o ausencia de la etiqueta con el sello verde en el empaque del producto. Esta etiqueta aparece como: "Producto Ecológico" sobre un fondo verde acompañado de un árbol, en una de las esquinas superiores de la respectiva imagen del producto. Se espera que los productos con etiqueta "ecológica" sean preferidos más que aquellos que no la tienen.

La tercera variable consiste en el tiempo de exposición a las alternativas, para lo cual se han diferenciado dos períodos de exposición: 60 segundos y 30 segundos. De acuerdo con lo propuesto por Logue (1995), se ha planteado la hipótesis de que los sujetos que han estado expuestos durante un período mayor (60 segundos) elegirán con mayor probabilidad la alternativa ecológica frente a aquellos sujetos que han tenido un período de exposición breve (30 segundos).
Para la totalidad del experimento se utilizaron 120 personas, $67 \mathrm{mu}$ jeres y 53 hombres, con una edad promedio de 26,1 años. El perfil de los participantes comprendió, en su mayoría, estudiantes universitarios de pregrado, profesores universitarios y empleados de una compañia de televisión satelital, todos residentes en la ciudad de Bogotá D.C. 
Finalmente, para la variable precio se han establecido tres condiciones para la comparación de las marcas A y B, las cuales se describen en la definición de variables. Específicamente, para las condiciones de precio mayor o menor, se determinó que para las categorías de producto con un precio mayor (por ejemplo \$900.000), el descuento sea del $20 \%$ aproximadamente, con el fin de ajustarse a la Ley de Weber, la cual establece que a medida que un precio es mayor, se requiere una diferencia cada vez más grande para que el consumidor perciba que en realidad existe una diferencia significativa entre dos precios. Para las categorías con un precio menor (por ejemplo, \$3.500), el descuento será del 10\% para ajustarse a la mencionada ley de percepción de Weber, ya que con este nivel de precios un descuento del $20 \%$ sesgaría demasiado la respuesta de los participantes. Con esta manipulación se deseaba hallar la interacción que tiene el precio con las otras variables para saber bajo qué condiciones es más probable que la alternativa ecológica sea elegida frente a la opción no ecológica.
Para la creación de las situaciones de elección y sus correspondientes alternativas, se consultó categorías de productos ecológicos de recientes diseños en países desarrollados, con el fin de indagar las preferencias del consumidor colombiano frente a estas opciones. Las categorías fueron escogidas por su relevancia de acuerdo con lo reportado por Ottman (2001) adaptándolas a un formato de elección en la pantalla del computador. La información utilizada para diseñar cada una de las condiciones aparece detallada en el anexo 1.

Muestra: Para la totalidad del experimento se utilizaron 120 personas, $67 \mathrm{mu}$ jeres y 53 hombres, con una edad promedio de 26,1 años. El perfil de los participantes comprendió, en su mayoría, estudiantes universitarios de pregrado, profesores universitarios y empleados de una compañía de televisión satelital, todos residentes en la ciudad de Bogotá D.C. Para la escogencia de los participantes no se establecieron variables de control, como, por ejemplo, la edad, el nivel socioeconómico o el nivel educativo.

Tabla No.1

Diseño factorial $3 \times 2 \times 2$ anidado en la variable "información de consecuencias de la decisión"

\begin{tabular}{|c|c|c|c|c|c|}
\hline & & \multicolumn{4}{|c|}{ Sin información de consecuencias } \\
\hline \multirow[t]{5}{*}{ PRECIO } & & \multicolumn{2}{|c|}{ Con etiqueta } & \multicolumn{2}{|l|}{ Sin etiqueta } \\
\hline & & 60 seg. & 30 seg. & 60 seg. & 30 seg. \\
\hline & $A=B$ & 10 Sujetos & 10 Sujetos & 10 Sujetos & 10 Sujetos \\
\hline & $A>B$ & 10 Sujetos & 10 Sujetos & 10 Sujetos & 10 Sujetos \\
\hline & $A<B$ & 10 Sujetos & 10 Sujetos & 10 Sujetos & 10 Sujetos \\
\hline & & Con informa & $\mathrm{n}$ de consec & cias & \\
\hline & & Con etiquet & & Sin etiqueta & \\
\hline \multirow[t]{4}{*}{ PRECIO } & & 60 seg. & 30 seg. & 60 seg. & 30 seg. \\
\hline & $A=B$ & 10 Sujetos & 10 Sujetos & 10 Sujetos & 10 Sujetos \\
\hline & $A>B$ & 10 Sujetos & 10 Sujetos & 10 Sujetos & 10 Sujetos \\
\hline & $A<B$ & 10 Sujetos & 10 Sujetos & 10 Sujetos & 10 Sujetos \\
\hline
\end{tabular}


Anexo 1

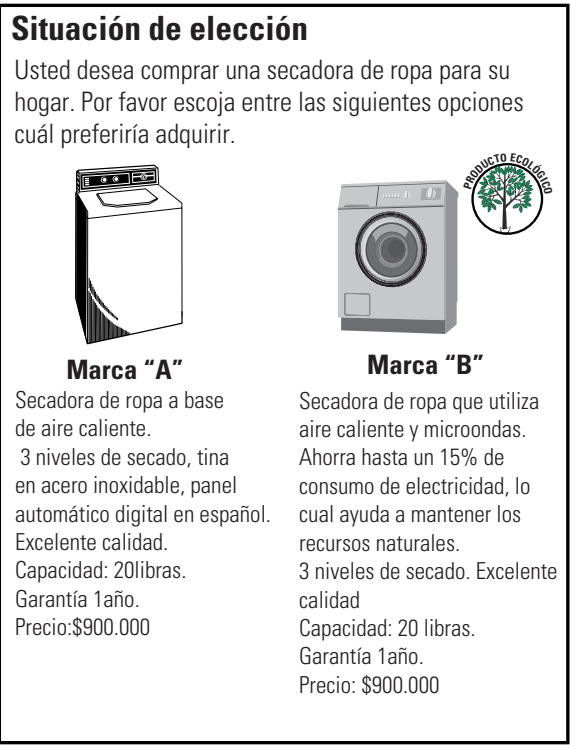

Recolección de datos: Cada sujeto se ubicó frente a una terminal de computadora y se le suministró las respectivas instrucciones para responder. Se les explicó la naturaleza del estudio, es decir, que era una compra simulada y que debían realizar 20 compras de productos, 10 por cada una de las fases del estudio. Cada persona eligió una alternativa a través del teclado del computador, marcando la tecla "Z" si prefería comprar la "marca A", que se encuentra a la izquierda, o marcando la tecla $M$ si prefería comprar la "marca B", que está a la derecha. Esta respuesta fue registrada automáticamente en el sistema. En cada condición, el tiempo para responder estuvo programado automáticamente y apareció en la parte superior derecha a manera de cronómetro, mostrando el tiempo que quedaba disponible para realizar la elección. Entre las aplicaciones de las fases uno y dos, a los sujetos se les dio 5 minutos de descanso para que realizaran actividades de distracción, como leer, hablar o trabajar en otro programa, con el fin de disminuir la interferencia de la información entre las dos fases.

Materiales: Los materiales consistieron en un programa informático especialmente diseñado para esta investigación denominado Ecochoice $2003^{\mathrm{TM}}$, el cual fue instalado en una serie de computadores dentro del centro de cómputo de una prestigiosa universidad ubicada en la ciudad de Bogotá D.C. En él estaban programados los diferentes elementos constitutivos del experimento los cuales incluían desde un cuadro de diálogo para introducir las variables de identificación de los individuos como las imágenes computarizadas que mostraban los estímulos necesarios para la simulación. La primera diapositiva que apareció en la pantalla mostraba un resumen del experimento, instruyendo a los sujetos acerca de su participación en una simulación de compra para una serie de productos e informándoles además que los nombres de las dos marcas serían reemplazados por las etiquetas A y B.

Para cada una de las situaciones de elección, se le presentó a los sujetos una imagen en la pantalla con las dos marcas de producto (ecológico y normal) y sus respectivos precios y características técnicas. Después de observar cada imagen, los sujetos utilizaron el teclado del computador para escoger por medio de dos teclas diferentes la marca que comprarían. Estos datos se constituyeron en las mediciones dependientes.

Las imágenes con los productos fueron precedidas por tres imágenes instructivas, en las cuales se hicieron tres ejercicios de hay un efecto significativo del tiempo de exposición sobre la elección realizada, de tal forma que los sujetos que tuvieron 30 segundos como tiempo máximo para elegir, escogieron consistentemente más la alternativa no ecológica, 
prueba para que las personas se adaptaran al mecanismo de respuesta y elección. Una muestra de las situaciones de elección y de todos los productos utilizados en el estudio aparece en el anexo 2.

\section{RESULTADOS}

Para el análisis de los resultados se aplicaron las siguientes pruebas estadísticas:

En primer lugar, la prueba Ji cuadrado entre cada factor y la variable dependiente. Con esta prueba se pretende hallar la interacción existente entre cada una de las condiciones de las variables independientes y las alternativas de elección (ecológica y no ecológica) para cada una de las situaciones de compra. En segundo lugar se elaboró un análisis del modelo lineal multivariante con el fin de hallar el nivel en el cual las variables independientes explican la variación en las respuestas de elección. Se pretende identificar, además, un posible modelo multivariado que pueda llegar a predecir con una significación estadística las preferencias de los consumidores frente a estas situaciones de compra. (Ver tabla 1).

En primer lugar, se encontró una diferencia significativa en la elección de la alternativa ecológica entre las dos condiciones de información $(\mathrm{p}<0,002)$ para la compra de la secadora, del detergente $(\mathrm{p}<0,000)$, del insecticida $(\mathrm{p}<0,024)$, del edredón $(\mathrm{p}<0,000)$ y de las esponjillas $(\mathrm{p}<0,001)$. De esta forma se confirmó la hipótesis con respecto a que los sujetos escogieron significativamente más la alternativa ecológica durante la fase de mayor información sobre las consecuencias de elección.

Para la categoría de esferos ( $\mathrm{p}<0,083)$, lavadora $(p<0,060)$ y papel $(p<0,062)$, aunque la prueba no alcanza a arrojar diferen-

Anexo 2

\section{Situación de elección}

Usted desea comprar una resma de papel. Por favor escoja entre las siguientes opciones cuál preferiría adquirir.

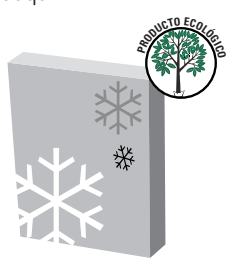

Marca "A"

Resma de papel blanco. Contenido: 500 hojas

tamaño carta.

Papel hecho de bambú, ya que esta especie vegetal permite

ahorrar energía y productos

químicos durante la producción del papel.

Digital, alta blancura, calidad superior, excelente desempeño para impresiones y copias.

Precio: $\$ 8.500$

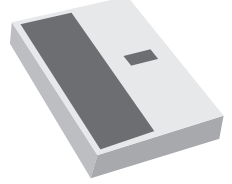

Marca "B"

Resma de papel blanco Contenido:500 hojas tamaño carta.

Papel hecho de pulpa de árbol proveniente de bosques tropicales.

Digital, alta blancura, calidad superior, excelente desempeño para impresiones y copias. Precio: $\$ 8.500$

cias significativas, sí se nota una tendencia a modificar la elección a favor de la marca ecológica en presencia de información.

En general, no se encontraron diferencias significativas entre el factor etiqueta y la elección realizada por los sujetos. Al analizar el factor tiempo, se encuentra que en la situación de compra de desechables $(\mathrm{p}<0.090)$, esferos $(\mathrm{p}<0,083)$, esponjillas $(\mathrm{p}<0,052)$ y lavadora $(\mathrm{p}<0,060)$, existe una tendencia a elegir significativamente más la marca ecológica cuando hay mayor tiempo de exposición a las alternativas, sin que alcancen a darse diferencias estadísticamente significativas. Para las situaciones de compra de la secadora $(\mathrm{p}<0,006)$ y el papel $(\mathrm{p}<0,034)$ hay un efecto significativo del tiempo de exposición sobre la elección realizada, de tal forma que los sujetos que tuvieron 30 segundos como tiempo máximo para elegir, escogieron consistentemente más la alternativa no ecológica, mientras que las personas que tuvieron 60 segundos 
Tabla 2. Valores de ji-cuadrado por categoría de producto

\begin{tabular}{l|cccc}
\hline & \multicolumn{4}{|c}{ NIVEL DE SIGNIFICACION PARA CADA VARIABLE } \\
INDEPENDIENTE \\
\hline CATEGORÍA & INFORMACIÓN & PRECIO & ETIQUETA & TIEMPO \\
\hline Secadora &, $002^{*}$ &, 515 &, 500 &, $006^{*}$ \\
\hline Detergente &, $000^{*}$ &, 433 &, 292 &, 180 \\
\hline Insecticida &, $024^{*}$ &, $002^{*}$ &, 136 &, 232 \\
\hline Edredón &, $000^{*}$ &, $031^{*}$ &, 305 &, 233 \\
\hline Esponjillas &, $001^{*}$ &, 372 &, 169 &, 052 \\
\hline Esferos &, 083 &, 627 &, 425 &, 083 \\
\hline Lavadora &, 060 &, 124 &, 169 &, 060 \\
\hline Papel &, 062 &, 624 &, 352 &, $034^{*}$ \\
\hline Desechables &, 107 &, 670 &, 264 &, 090 \\
\hline Muebles &, 531 &, 822 &, 060 &, 253
\end{tabular}

${ }^{*}$ Representa una diferencia estadísticamente significativa con un valor $p<0,05$. A partir del análisis de las tablas Ji cuadrado (Ver tabla 1) se encontró que sólo una de las variables independientes reflejó una diferencia estadísticamente significativa entre las dos condiciones experimentales.

para responder eligieron con mayor probabilidad la alternativa ecológica. Para estas dos categorías se confirmo la hipótesis planteada con respecto a esta variable.

En cuanto al factor precio, se encontraron diferencias significativas en la categoría edredón ( $\mathrm{p}<0,031$ ), en lo cual la marca ecológica se prefirió más que la no ecológica en las tres condiciones de precio. Esto pudo deberse a una aparente percepción de bajo precio frente a las características antialérgicas del edredón. En la categoría de insecticida también hay diferencias ( $\mathrm{p}<0,002)$, en donde la alternativa ecológica se prefiere en las condiciones de precio igual y precio de la marca ecológica menor al de la no ecológica, mientras que se prefiere más la no ecológica cuando ésta tiene un precio menor frente a la ecológica. Esto concuerda con la hipótesis planteada con respecto al precio, en la medida en que, si la marca ecológica es más costosa, será menos preferida que la no ecológica.

Sin embargo, para el resto de categorías no se halló ninguna diferencia significativa para la variable precio. Es probable que para la elección hayan influido de manera más decisiva los otros factores.

Para confirmar los resultados hallados anteriormente se realizó un análisis de regresión múltiple para determinar, al mismo tiempo, la influencia de cada una de las variables independientes sobre la elección. El análisis de la tabla del modelo multivariado que indica la influencia de los cuatro factores del estudio (etiqueta, información, precio y tiempo) en la variación de las respuestas de elección de los sujetos, indica que el factor información influye de manera significativa en la variación de las respuestas dadas por los participantes entre la primera y la segunda fase del estudio. Esto confirma la hipótesis con respecto a que en presencia de una información clara acerca de las consecuencias de adquirir el producto ecológico, el consumidor escogerá con mayor probabilidad esta alternativa frente a la alternativa impulsiva.

En cuanto a los otros factores, se encuentra que el tiempo y el precio, aunque no alcanzan a influir de manera significativa, sí muestran una fuerte tendencia a explicar 
parte de la variación de las respuestas de los sujetos ante las alternativas de elección. Al observar las interacciones entre factores se encuentra una relación importante entre el precio y la etiqueta, lo cual indica que en algunos casos la etiqueta ecológica ayuda a reforzar este atributo del producto cuando la marca ecológica tiene un precio menor al de su contraparte.

\section{DISCUSIÓN}

De acuerdo con los resultados hallados se encuentra que las conductas de elección de los sujetos se dirigen mayoritariamente hacia la alternativa de autocontrol, la cual proporciona una consecuencia más grande y demorada sobre la alternativa que ofrece una consecuencia más pequeña e inmediata, que en este caso correspondió a los productos no ecológicos. Este resultado fue en gran parte inesperado, ya que en otras investigaciones sobre autocontrol la preferencia de los sujetos se dirige hacia la alternativa impulsiva (Kivetz y Simonson, 2002).

Esto demuestra que para la muestra escogida las alternativas ecológicas son una opción con mayor valor, si se tiene en cuenta que bajo las diferentes combinaciones de variables las marcas ecológicas fueron más preferidas frente a las alternativas tradicionales. Estos resultados soportan el planteamiento de la Teoría de Limitación Temporal, ya que las consecuencias de la elección de las marcas ecológicas son más difusas a nivel psicológico, debido a que es difícil asegurar cuándo se va a dar un cambio positivo a nivel ambiental que se derive de las compras de estos productos. Por lo tanto, la elección de las alternativas ecológicas (menos con- cretas) fue mayor debido a que los sujetos escogieron el curso de acción que concordaba más con sus preferencias globales, en este caso, la protección del ambiente de las consecuencias nocivas de los productos no ecológicos. Este carácter difuso corresponde también a una situación de ambivalencia compleja, ya que las consecuencias de la alternativa ecológica son abstractas y difíciles de percibir en el corto plazo para el consumidor típico, sumado a una aparición temporal no definida de la consecuencia.

Se debe destacar el fuerte impacto ejercido por la presencia de información durante la segunda fase del estudio en el incremento y la preferencia por estas marcas ecológicas. Este hallazgo es promisorio, ya que demuestra que existe una actitud favorable hacia estos nuevos productos en una muestra de consumidores colombianos, que tienen unos valores culturales diferentes a los de los consumidores europeos, por ejemplo, quienes están más familiarizados con este tipo de productos. Como lo sugieren los resultados hallados, la variable informacional tiene un gran peso en el proceso de decisión de los consumidores, razón por la cual se le debe asignar una mayor importancia dentro de los esfuerzos realizados por las diferentes organizaciones que luchan por la defensa del medio ambiente, así como por las entidades gubernamentales responsables de estos asuntos. Si esta comunicación sobre las consecuencias de las elecciones se hace de manera efectiva, se podrían generar las metacontingencias que a largo plazo permitan la modificación de las prácticas culturales poco responsables con el medio ambiente por unas prácticas 
más autocontroladas, como por ejemplo, la adopción de conductas de reciclaje, la compra de productos con sello verde, entre otras.

En cuanto a la hipótesis relacionada con la elección de los bienes que implican una mayor duración en su consumo, se confirmó que existe una mayor preferencia por las marcas ecológicas en aquellos productos que implican este tipo de consumo extendido, como lo son la lavadora y la secadora.

Por otra parte, retomando el concepto de ambivalencia compleja propuesto por Rachlin (2000), se encontró que los sujetos experimentaron una disminución en la ambivalencia, ya que, de acuerdo con su marcada preferencia por los productos ecológicos, se concluye que lograron percibir esa consecuencia ambiental positiva, la cual es difusa en términos temporales y en términos de efectos concretos, gracias a la información proporcionada durante la segunda fase del estudio. Como se desprende de esto, sería conveniente incluir más información en las comunicaciones de mercadotecnia de este tipo de productos que hagan ver al consumidor una consecuencia concreta y real que generaría su conducta de elección ecológica.

En otras palabras, para reducir la ambivalencia se recomienda dar más información que haga que la consecuencia que es más positiva a largo plazo se vea de forma más concreta para el consumidor, sobre todo para aquellos que no tienen un buen nivel de conocimiento de los problemas planetarios a nivel ambiental. Sin embargo, sería recomendable hacia el futuro realizar estudios que hagan un seguimiento a las conductas de elección de los sujetos durante períodos de tiempo mayores, con el fin de determinar los niveles de inconsistencia de las elecciones teniendo en cuenta las alternativas ecológicas y no ecológicas.

$\mathrm{Al}$ analizar los cuatro factores en forma conjunta, se encontró que el modelo multivariado no explica de forma significativa la variación de las respuestas de elección, lo cual implica que en el proceso deben mediar otras variables que no fueron manipuladas aquí. Más adelante se sugieren algunas opciones al respecto, con el fin de que otras investigaciones puedan abordar el problema de una forma más amplia.

Finalmente, la investigación permitió identificar de forma clara que la información sobre las consecuencias de elección sí influyen en el proceso de decisión de los consumidores cuando se comparan dentro de una categoría de producto dos marcas, una ecológica y otra marca tradicional sin este tipo de beneficios ambientales. Este es un indicador claro de que en presencia de información, la población podría llegar a modificar sus hábitos de consumo en favor de productos que protejan el medio ambiente y generen un beneficio a largo plazo para toda la humanidad. Es por esto recomendable para los fabricantes proveer de información al consumidor desde este momento para que el proceso de cambio en la valoración de las marcas ecológicas permita una introducción al mercado más favorable, y pensar en la competencia que tendrían con productos tradicionales y probablemente con un costo menor.

Para los investigadores interesados en el tema, se recomienda realizar estudios que coloquen a los sujetos en situaciones de
Se confirmó que existe una mayor preferencia por las marcas ecológicas en aquellos productos que implican este tipo de consumo extendido, como lo son la lavadora y la secadora. 
elección dentro de circunstancias con mayor realismo, como es el caso de las estanterías experimentales en las cuales las personas observan, comparan y deciden directamente frente a las góndolas de un supermercado. Esto permitiría establecer si los productos ecológicos son en realidad más preferidos que los no ecológicos en diferentes condiciones, como por ejemplo con precios diferentes, diferentes cantidades de información, con o sin etiqueta, y en condiciones de compra con límite de tiempo, entre otros factores.

También se recomienda estratificar la muestra de acuerdo principalmente con el nivel de escolaridad y de ingresos. Con esto se lograría estudiar diferentes segmentos de consumidores con el fin de entender con mayor profundidad los procesos de elección dentro de esta categoría de producto

\section{BIBLIOGRÁFIA}

Ainslie, G (1975). “Specious reward: A behavioural theory of impulsiveness and impulse control", Psychology Bulletin, 82, April, 463-496.

Baumeister, R. (2002). "Yielding to temptation: Self-Control Failure, Impulsive purchasing and Consumer Behavior". Journal of Consumer Research, 28, March, 25-30.

Hernández, R., Fernández, C. y Baptista, P., (1991). Metodología de la Investigación. México D.F.: McGraw Hill Interamericana.

Hoch, S., Loewenstein, G. (1991). “Timeinconsistent Preferences and Consumer Self-Control”. Journal of Consumer Research, 17, March, 492-508.

Hull, J. (1981). “A Self-Awareness Model of the Causes and Effects of Alcohol
Consumption”. Journal of Abnormal Psychology. 90, December, 586-600.

Kivetz, R., Simonson, I. (2002). "Self-

Control for the Righteous: Toward a Theory of Precommitment to Indulgence". Journal of Consumer Research, 29, September, 281-305.

Liberman, N. y Trope, Y. (1998). “The role of feasibility and desirability considerations in near and distant future decisions: A test of Temporal Construal Theory". Journal of personality and Social Psychology. 75, July, 5-18.

Logue, A.W. (1995). Self-control, New York: Prentice Hall.

_. (1996). "Self-control: An alternative self-regulation framework applicable to human and nonhuman behavior". Psychological Inquiry, 7, (1), 50-68.

Marting, B. (2002). Guía para la elaboración y presentación de trabajos de investigación. Bogotá D.C.: Ediciones Ibáñez.

Mowen, J. (1998). Consumer Behavior. New York: Prentice Hall.

Muraven, M., Baumeister, R., Tice, D. (1994). "Longitudinal improvement of self-regulation through repeated exercise". Journal of Social Psychology, 139, (4), 432-446.

Nebel, B. (1999). Ciencias Ambientales: Ecología y Desarrollo Sostenible. México D.F.: Pearson Educación.

O’Guinn, T., Faber, R. (1989). “Compulsive Buying: A Phenomenological Exploration". Journal of Consumer Research, 16, September, 147-157.

Ottman J. (2001). Green Marketing: Opportunity for Innovation, New York: Mc Graw Hill.

Rachlin, H. (2000). The Science of Self- 
Control. New York: University of Stony Brook.

Roper International Research Associates, (1995). Green Gauge.

Schelling, T. (1984). "Self-Command in practice, in policy, and in a theory of rational choice". American Economics Association Proceeds , 74 May, 1-11.
Solomon, M. y Stuart, E. (2001), Marketing: Personas reales, decisiones reales. Bogotá D.C.: Prentice Hall.

Thaler, R., Shefrin, H. (1981). "An economic theory of Self-Control". Journal of Political Economy, 89 , April, 392410. 